\title{
Initiatorless Photopolymerization of Liquid Crystal
}

\section{Monomers}

Kyung Min Lee, ${ }^{\dagger}$ Taylor H. Ware, ${ }^{\dagger \S}$ Vincent P. Tondiglia, ${ }^{\dagger}$ Matthew K. McBride, ${ }^{\#}$ Xinpeng

Zhang, ${ }^{\#}$ Christopher N. Bowman, ${ }^{\#}$ and Timothy J. White*广

$\dagger$

Air Force Research Laboratory, Materials and Manufacturing Directorate, Wright-Patterson Air Force Base, Ohio 45433-7750, United States.

$\$$ Azimuth Corporation, 4027 Colonel Glenn Hwy, Beavercreek, Ohio 45431, United States.

${ }^{\S}$ Department of Bioengineering, The University of Texas at Dallas, Richardson, Texas 75080, United States.

\# Chemical and Biological Engineering, University of Colorado, Boulder, Colorado 80309, United States.

*Email: Timothy.White.24@us.af.mil 
Supporting Information

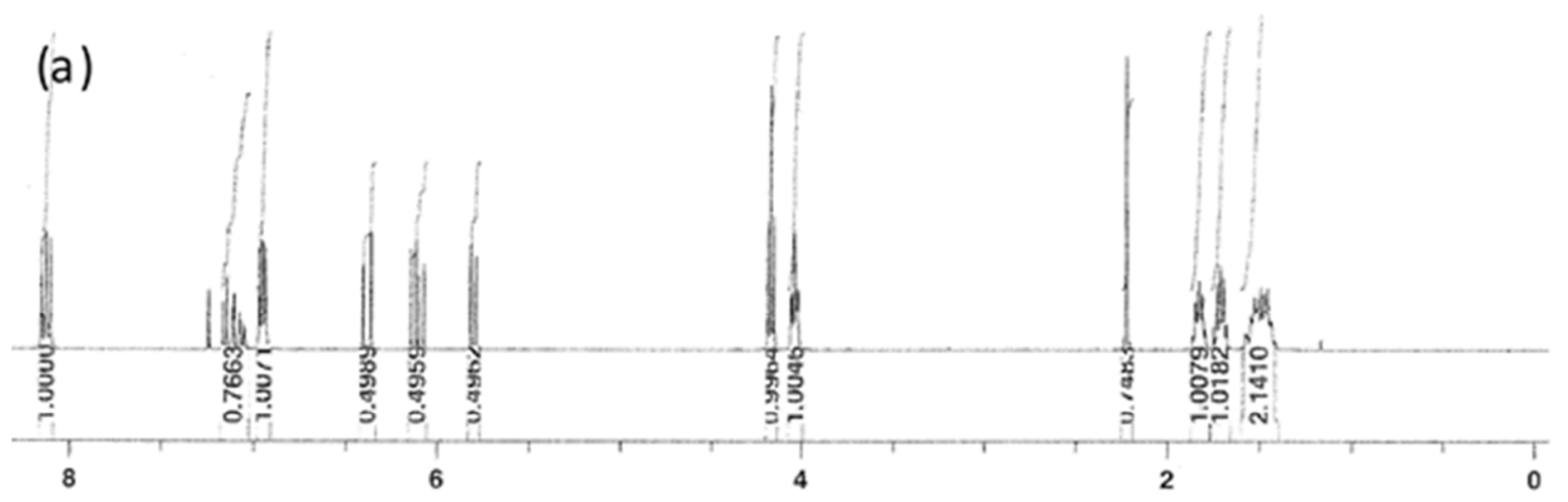

(b)

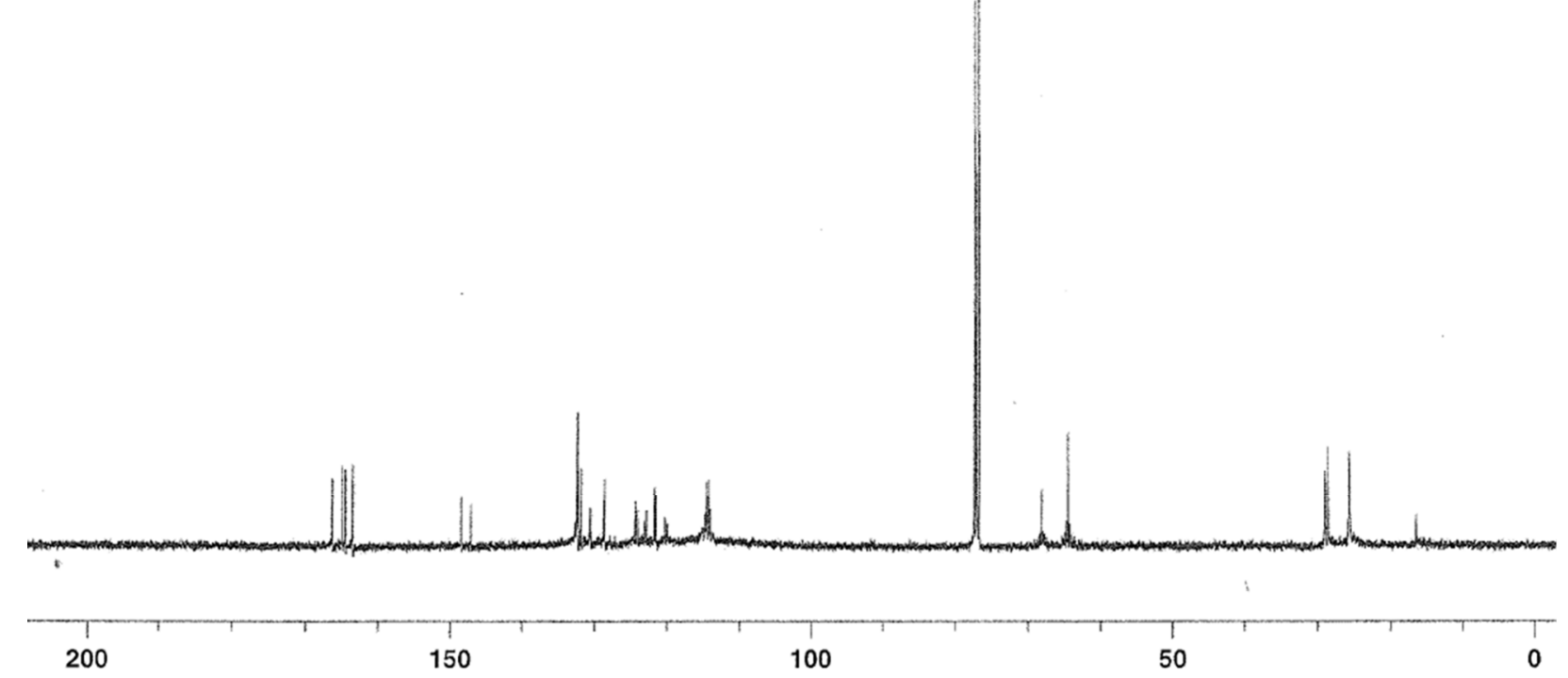

Figure S1. (a) 1H and (b) 13C NMR spectroscopes of RM82. 
Supporting Information

\begin{tabular}{|l|l|l|l|l|l|l|}
\hline \multirow{2}{*}{ Samples } & \multicolumn{2}{|l}{ C } & \multicolumn{2}{l}{ H } & \multicolumn{2}{l|}{ O } \\
\cline { 2 - 7 } & Theory & $\%$ found & Theory & $\%$ found & Theory & $\%$ found \\
\hline RM82 & 69.63 & 69.55 & 6.59 & 6.55 & 23.78 & 23.98 \\
\hline RM257 & 67.34 & 66.97 & 5.48 & 5.58 & 27.18 & 26.92 \\
\hline
\end{tabular}

Nitrogen is not found.

Table S1. Results of elemental analysis of two liquid crystal monomers. Tested by MIDWEST MICROLAB, Inc.

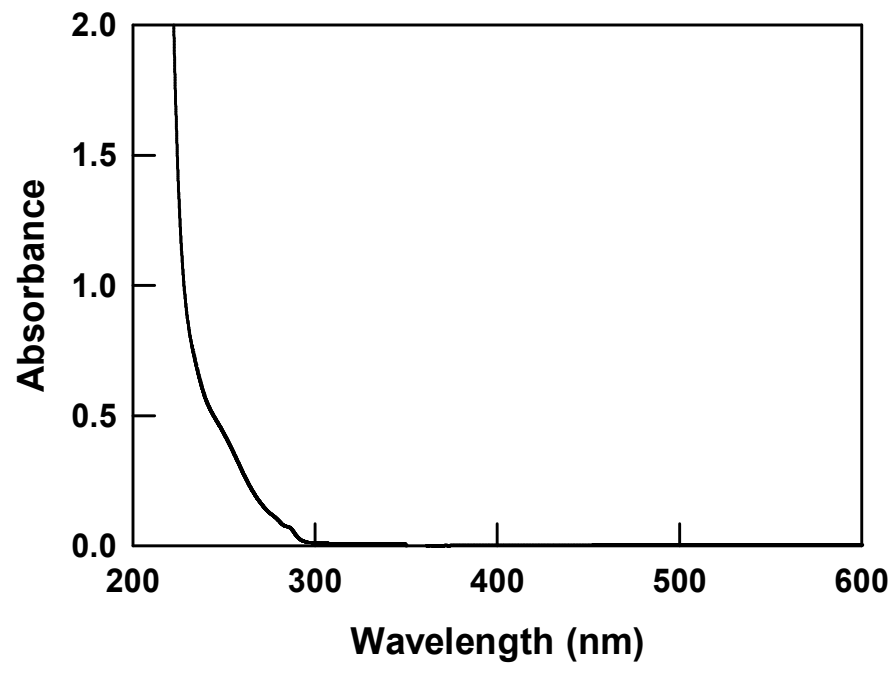

Figure S2. UV/Vis absorption spectrum of the 1,6-hexanediol diacrylate (HDDA). 
Supporting Information

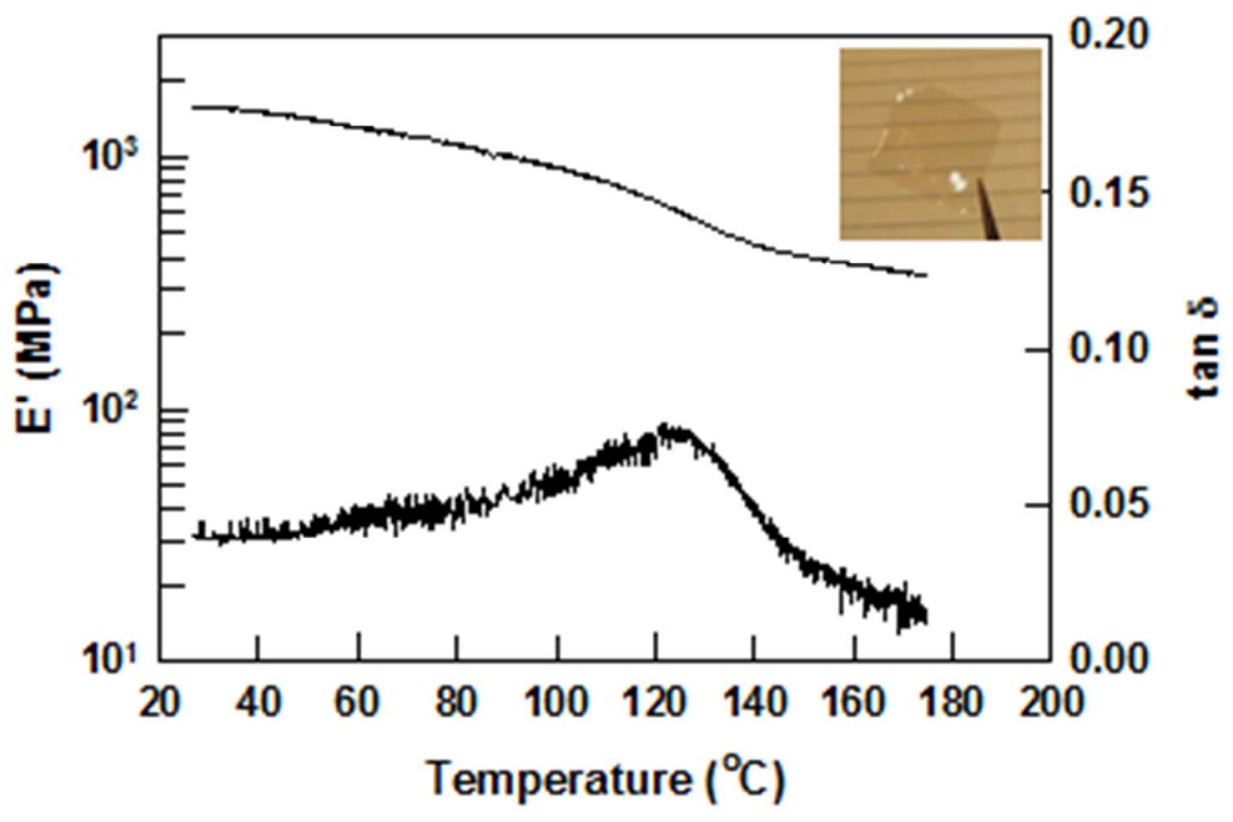

\begin{tabular}{|l|c|c|c|}
\hline Sample & I-369 concentration $(w t \%)$ & $E^{\prime}(\mathrm{GPa})$ at $30^{\circ} \mathrm{C}$ & $\operatorname{Tan} \delta\left({ }^{\circ} \mathrm{C}\right)$ \\
\hline Poly(RM82)-0 & 0 & 1.5 & 117 \\
\hline Poly(RM82)-2 & 2 & 1.6 & 125 \\
\hline
\end{tabular}

Figure S3. DMA results of Poly(RM82) samples prepared with or without photoinitiator at the exposure of $50 \mathrm{~mW} / \mathrm{cm}^{2} 365 \mathrm{~nm} \mathrm{UV}$ for $1 \mathrm{~h}$. 
Supporting Information

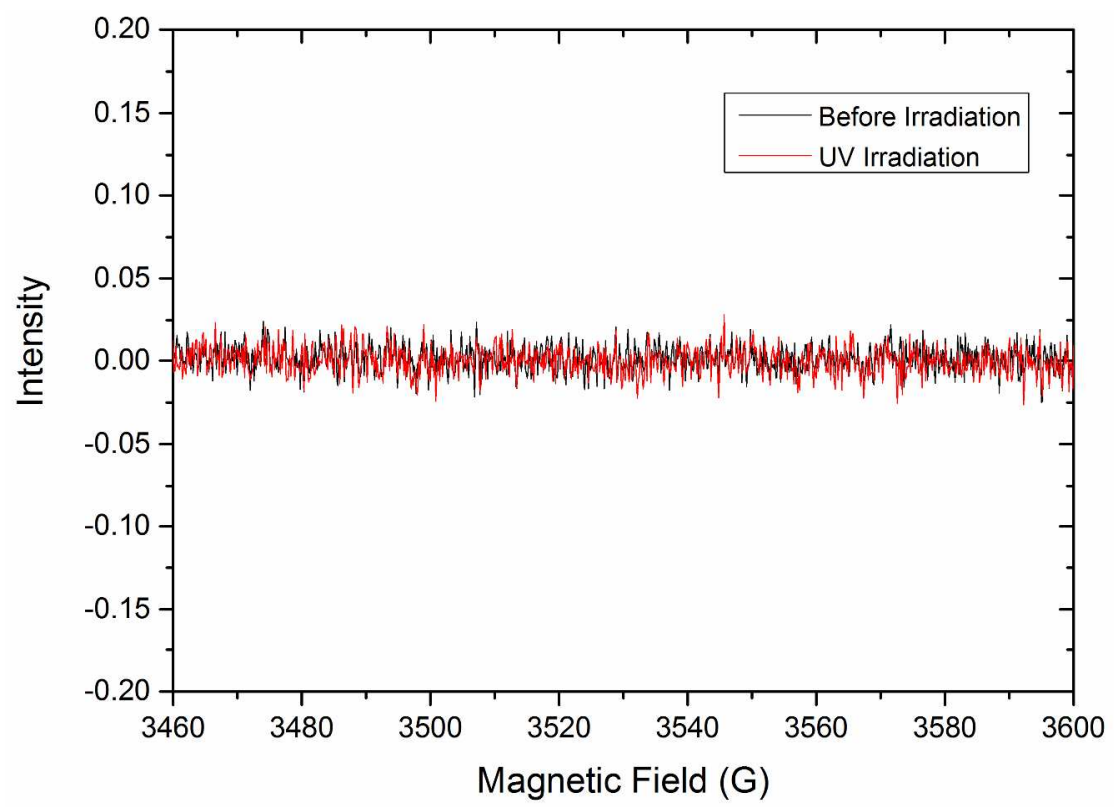

Figure S4. EPR results for $6 w t \%$ RM82 solution in THF before and after UV irradiation (50 $\left.(+/-5) \mathrm{mW} / \mathrm{cm}^{2}\right)$. 